\title{
P-Wave Reflection Approximation of a Thin Bed and Its Application
}

\author{
Chun Yang *, Yun Wang, Shu Xiong, Zikun Li® and Hewei Han
}

School of Geophysics and Information Technology, China University of Geosciences, Beijing 100083, China; yunwang@mail.iggcas.ac.cn (Y.W.); 3010190020@cugb.edu.cn (S.X.); 2010180020@cugb.edu.cn (Z.L.); 2010180058@cugb.edu.cn (H.H.)

* Correspondence: yangchun@cugb.edu.cn

Received: 17 October 2020; Accepted: 12 November 2020; Published: 13 November 2020

\begin{abstract}
Thin-bed" reservoirs have become important targets of seismic exploration and exploitation. However, traditional amplitude versus offset/amplitude versus angle (AVO/AVA) technologies, for example, those based on Zoeppritz equations and their approximations for a single interface, are not sufficiently accurate for thin-bed stratigraphy. Analytic solutions of thin-bed reflectivity may become practical for thin-bed AVO analysis and inversion. Therefore, a linear analytic approximation of thin-bed P-wave reflectivity is developed under small-incidence and thin-bed assumptions. Numerical simulations show that the amplitude approximation errors are usually smaller than $10 \%$ for incidence angles less than 20 degrees, and the thin-bed thicknesses are less than one-tenth of the P-wave wavelength. Based on the least-squares approach, the inversion strategy is proposed using the approximate formula. A synthetic data test shows that the proposed inversion method can produce more accurate thin-bed properties than that based on the Zoeppritz equations, which reveals the potential of the inversion method based on the linear analytic approximate formula in the fine characterization of thin reservoirs.
\end{abstract}

Keywords: thin bed; P-wave response; approximated formula; application

\section{Introduction}

A large percentage of oil and gas reservoirs exist as thin beds, as their thicknesses are below seismic resolution [1]. Studies show that thin-bed amplitude versus offset/amplitude versus angle (AVO/AVA) responses depend on the thin-bed thickness and may differ significantly from that of a single interface [2,3]. Therefore, traditional AVO/AVA analysis and inversion methods, which are based on Zoeppritz equations for a single interface, are ineffective for thin-bed seismic simulation and inversion. The main problem associated with thin beds is the interference effects of all the reflecting waveforms, including converted waves and multiples.

Thin-bed reflection/transmission (R/T) coefficients in isotropic media have been studied for several decades, with discussions mainly focused on the relationship between thin-bed reflections and thickness [4-10]. For the cases of arbitrary incidence, Thomson [11], Haskell [12] and Brekhovskikh [13] studied reflections and transmissions of layered elastic media and provided solutions with matrix propagation. However, the complexity of their propagator matrix limited its application. Meissner and Meixner [14] and Juhlin and Young [3] presented a time-delayed R/T method and deduced that thin-bed reflections were the total stack responses of primary reflections at the top interface and the intra-bed multiples time-delayed to the primary reflection. Yang et al. [15] gave three types of approximate analytical formulas for thin-bed PP-wave reflectivity based on the work of Juhlin and Young [3] by ignoring converted waves and intra-bed multiples. Kennett et al. [16] and Kennett [17] built up the reflection and transmission matrices recursively by starting at the top interface and working 
down through the successive interfaces iteratively. Liu and Schmitt [10] gave an analytical formula of thin-bed seismic responses in acoustic media by ignoring multiple waves. Rubino and Velis [18] extended the work of Liu and Schmitt [10] into elastic media and used amplitude spectra to achieve thin-bed pre-stack spectral inversion. Pan and Kristopher [19] utilized Brekhovskikh's layered elastic medium theory to develop a three-layer case and pointed out that thin-bed effects are significantly different in elastic media than in acoustic media, especially for small incidence angles.

The authors mentioned above gave a clear description of thin-bed responses. However, their thin-bed R/T coefficient formulas for arbitrary incidence are generally too complex for applications or have insufficient accuracy in that they disregarded the effects of thin-bed intra-bed multiples and converted waves. Yang et al. [20] gave thin-bed true R/T coefficients defined by displacement and approximated them to be in a quasi-Zoeppritz matrix form under the thin-bed assumption. After that, they [21] gave second-order series approximation for PP-wave reflection of a thin bed and estimated thin-bed properties, including a P-wave impedance ratio and thickness by statistical data. Considering that analytic approximations of reflection coefficients are important in AVO analysis and inversion for a single interface [22,23], we further simplify second-order series approximation under a thin-bed assumption for thin-bed AVO/AVA analysis and inversion in this paper.

First, we give a P-wave reflected approximation of a thin bed under a thin-bed assumption based on second-order series approximation. Then, we test the accuracy of the linear analytic approximation in this paper derived through numerical simulations of four representative thin-bed models. Finally, we exhibit the applicability of our approximate formula in thin-bed inversion of synthetic seismic data.

\section{Theory}

\subsection{P-Wave Reflected Approximation of a Thin Bed}

For a thin bed in elastic isotropic media, as shown in Figure 1, Yang et al. [21] gave second-order series approximation for P-wave reflection as follows,

$$
R_{P P} \approx A_{0}+A_{2} \sin ^{2} \theta,
$$

where $\theta$ is incidence angle, $A_{0}$ and $A_{2}$ are the intercept and gradient, respectively, which are presented in Appendix A in detail.

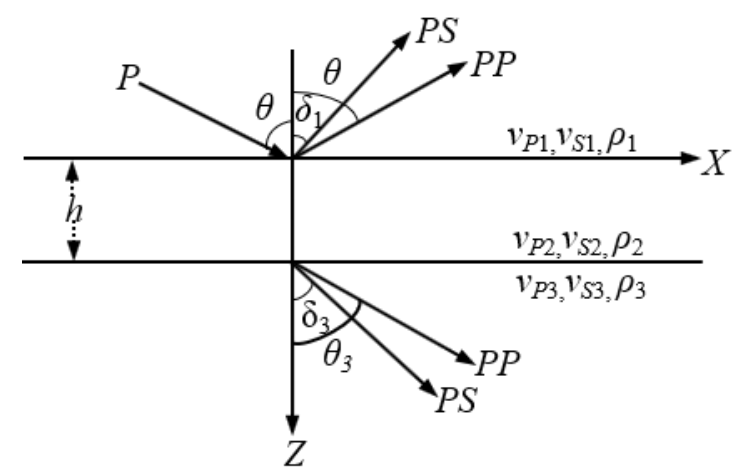

Figure 1. A thin-bed model with two horizontal interfaces. P is incident P-wave, PS is reflected or transmitted S-wave, PP is reflected or transmitted P-wave, other parameters are introduced in Appendix A [21]. 
When the middle layer's thickness $h$ is ultrathin, then $\omega h / v_{\mathrm{P} 2}<<1$ and $\omega h / v_{\mathrm{S} 2}<<1$, which are defined as $\tau$ and $q$, respectively, in Equation (1) (shown in Appendix A). That is, $\tau<<1$ and $q<<1$. Using first-order Taylor expansions,

$$
\sin \tau \approx \tau, \sin q \approx q, \cos \tau \approx 1, \cos q \approx 1,
$$

Equation (1) is simplified as

$$
R_{P P} \approx P+G \sin ^{2} \theta
$$

with

$$
\begin{gathered}
P=\frac{1}{n_{0}}\left(\frac{z_{P 3}}{z_{P 1}}-1\right)+\frac{j \tau}{n_{0}}\left(\frac{z_{P 2}}{z_{P 1}}-\frac{z_{P 3}}{z_{P 2}}\right), \\
G=\frac{1}{2 n_{0}} \quad\left(\frac{z_{P 3}}{z_{P 1}}+j \tau \frac{z_{P 2}}{z_{P 1}}+\frac{4 j \tau l_{1}}{r_{1}^{2}}\right)\left(P+\frac{2}{r_{1}} A-1\right)+\frac{l_{1} l_{2}}{n_{0} r_{3}}\left(1-\frac{2}{r_{3}}\right) \frac{z_{P 3}}{z_{P 1}} B \\
+\frac{1}{n_{0}}\left[\frac{2}{r_{1}^{2}}+j \tau\left(l_{1}-\frac{2 l_{1}}{r_{2}^{2}}+\frac{2}{r_{1}^{2}} \frac{z_{P 1}}{z_{P 2}}\right) \frac{z_{P 3}}{z_{P 1}}\right](1+P+A) \\
+\frac{j \tau l_{1}}{n_{0} r_{1}}\left(1-\frac{4}{r_{1}^{2}}\right) A+\frac{l_{1}^{2}}{n_{0}}\left(\frac{1}{2}-\frac{2}{r_{3}^{2}}\right) \frac{z_{P 3}}{z_{P 1}} \frac{2}{n_{0}}\left(1+\tau^{2}\right)
\end{gathered}
$$

Other parameters are presented in Appendix B in detail.

Equation (3) is the linear analytic approximation of a thin-bed P-wave reflection coefficient. The approximate formula is written as a sum of the constant term and the first order term of the sine-squared incidence angle. Compared with Equation (1), the linear analytic approximation has a relatively compact form (shown in Appendices A and B), which is more convenient to achieve AVO/AVA analysis and inversion of a thin bed.

Compared with linearized approximations to the Zoeppritz equations for a single interface [22-24], the intercept and gradient of the linear analytic approximation for the thin-bed P-wave reflectivity are frequency dependent; furthermore, they are functions of elastic parameters of the target thin bed and the upper and lower half spaces. Meanwhile, the linear analytic approximation is derived with no weak impedance contrast hypothesis, so it is valid for thin-bed models with strong impedance contrasts, such as coal seams, etc.

\subsection{Inversion of Thin-Bed Elastic Parameters and Thickness}

Based on the linear analytic approximation, we invert for elastic parameters and thickness of the thin-bed reservoir, including P- and S-wave velocities and densities of the target thin bed and its upper and lower half spaces and thin-bed thickness. The inversion procedure of thin-bed properties is as follows.

\subsubsection{Extraction of Complex Reflection Coefficients}

Because thin-bed reflection coefficients are complex valued and frequency dependent, we construct complex seismic trace and analyze it in the frequency domain. The conventional AVA seismic trace $\mathbf{s}^{\text {real }}(\theta, t)$ of a thin bed is viewed as the real component of thin-bed complex trace. The imaginary component $\mathbf{s}^{i m}(\theta, t)$ can be calculated through Hilbert transform [25-27]:

$$
\mathbf{s}^{i m}(\theta, t)=\mathbf{s}^{\text {real }}(\theta, t) * \frac{1}{\pi t}
$$

where the symbol * is time convolution.

The complex AVA trace $\mathbf{s}(\theta, t)$ in the time domain is:

$$
\mathbf{s}(\theta, t)=\mathbf{s}^{\text {real }}(\theta, t)+j \mathbf{s}^{i m}(\theta, t),
$$


where $j$ is the imaginary term. $\mathbf{s}(\theta, t)$ in the frequency domain can be obtained through Fourier transform:

$$
\mathbf{S}(\theta, f)=\operatorname{FFT}[\mathbf{s}(\theta, t)]
$$

If the seismic wavelet $\mathbf{w}(\theta, t)$ in the time domain is known, we can calculate its value in the frequency domain through Fourier transform:

$$
\mathbf{W}(\theta, f)=\operatorname{FFT}[\mathbf{w}(\theta, t)] .
$$

Thus, the reflection coefficients of a thin bed in the frequency domain can be derived by:

$$
\mathbf{R}^{o b s}(\theta, f)=\mathbf{S}(\theta, f) / \mathbf{W}(\theta, f),
$$

where $\mathbf{R}^{o b s}(\theta, f)$ is the observed reflection coefficient matrix of an AVA dataset at different frequencies.

If the seismic wavelet is not known, Liu et al. [28] gave a method to estimate the reflection coefficient from seismic data for a single-interface case. We can extend Liu's method to estimate $\mathbf{R}^{\text {obs }}$ by extracting its real and imaginary components from those of AVA traces, respectively.

\subsubsection{AVA Inversion of Thin-Bed Properties}

According to a least-squares approach, the objective function $Q$ for the inversion of the thin-bed P-wave reflection coefficient is defined as follows:

$$
Q(\mathbf{m})=\left\|\mathbf{R}^{o b s}-\mathbf{R}\right\|^{2},
$$

where $\mathbf{m}=\left(\mathbf{v}_{\mathrm{P}} \text { vs. } \boldsymbol{\rho} \mathrm{h}\right)^{\mathrm{T}}$ are the thin-bed elastic parameters and thickness. $\mathbf{R}$ is the vector of the calculated P-wave reflection coefficients by the linear analytic approximation for the given model parameter vector $\mathbf{m}$. The update model vector $\Delta \mathbf{m}=\left(\Delta \mathbf{v}_{\mathrm{P}} \Delta \mathbf{v}_{\mathrm{S}} \Delta \boldsymbol{\rho} \Delta \mathrm{h}\right)^{\mathrm{T}}$ can be calculated through the Gauss-Newton formula [29-32] as:

$$
\Delta \mathbf{m}=\left[\mathbf{J}^{T}(\mathbf{m}) \mathbf{J}(\mathbf{m})+\chi \mathbf{I}\right]^{-1} \mathbf{J}^{T}(\mathbf{m})\left[\mathbf{R}\left(\mathbf{m}_{0}+\Delta \mathbf{m}\right)-\mathbf{R}\left(\mathbf{m}_{0}\right)\right],
$$

where $\mathbf{m}_{0}$ is the initial parameter vector, $\chi$ is a Lagrange multiplier, $\mathbf{I}$ is an identity matrix and $\mathbf{J}$ is the Jacobian matrix as

$$
\mathbf{J}=\left[\frac{\partial \mathbf{R}_{i}(\mathbf{m})}{\partial m_{k}}\right] \text { with } i=1, \ldots, N A \times N F ; k=1, \ldots, N M,
$$

where the subscripts $i, k$ indicate indices for reflection coefficients and model parameters, respectively; $\mathrm{NA}, \mathrm{NF}$ and NM are the numbers of angle, frequency and model parameters, respectively.

Considering that $\mathbf{m}$ has many parameters, including $\mathrm{P}$-wave velocities, $\mathrm{S}$-wave velocities, densities of three layers and the thickness of middle layer for a thin bed, $t$ is more prone to obtaining local minimum solutions than conventional AVA inversion. In order to obtain reasonable inversion results, priori information is adopted to constraint the inverted thin-bed model.

\section{Numerical Analysis and Application}

\subsection{Approximation Accuracy Analysis}

In order to verify the accuracy of the linear analytic approximation of the thin-bed P-wave reflection coefficient derived in this paper, we conduct tests on four representative thin-bed models, including: (1) high-impedance thin bed, (2) low-to-high impedance transition layers, (3) low-impedance thin bed and (4) high-to-low impedance transition layers [10,33]. Table 1 lists the elastic parameters and Figure 2 plots the reflectivity logs of these four models. Figure 2 shows that Models 1 and 3 produce opposite-polarity reflections, while Models 2 and 4 produce identical-polarity reflections. Compared with Models 2-4, Model 1 has stronger impedance contrasts, which are utilized to test 
the approximate formula's applicability in thin-bed models with strong impedance contrasts. In the numerical simulations, we set thin-bed thicknesses as $\lambda / 8, \lambda / 10, \lambda / 20, \lambda / 30, \lambda / 40, \lambda / 50$ and $\lambda / 60$, where $\lambda$ is the P-wavelength in the target thin bed. Considering the small-incidence assumption, the incidence angle in all cases is set from 0 degree to 30 degrees with a 1-degree sample interval.

Table 1. Parameters of thin-bed models. $I_{P}$ and $I_{S}$ are P-wave and S-wave impedances, respectively, units of velocities and densities in Table 1 are $\mathrm{m} / \mathrm{s}$ and $\mathrm{g} / \mathrm{cm}^{3}$, respectively.

\begin{tabular}{ccccccc}
\hline & Layer No. & $\boldsymbol{v}_{\boldsymbol{P}}$ & $\boldsymbol{v}_{\boldsymbol{S}}$ & $\boldsymbol{\rho}$ & $\mathbf{I}_{\boldsymbol{P}}$ & $\mathbf{I}_{\mathbf{S}}$ \\
\hline \multirow{3}{*}{ Model 1 } & 1 & 3050 & 1525 & 2.7 & 8235 & 4117.5 \\
& 2 & 6100 & 3050 & 2.7 & 16,470 & 8235 \\
& 3 & 2500 & 1525 & 2.7 & 6750 & 4117.5 \\
\hline \multirow{3}{*}{ Model 2 } & 1 & 3050 & 1600 & 2.7 & 8235 & 4320 \\
& 2 & 4200 & 2500 & 2.7 & 11,340 & 6750 \\
& 3 & 6100 & 3100 & 2.7 & 16,470 & 8370 \\
\hline \multirow{3}{*}{ Model 3 } & 1 & 2200 & 1200 & 2.3 & 5060 & 2760 \\
& 2 & 1800 & 800 & 2.2 & 3960 & 1760 \\
& 3 & 3050 & 1400 & 2.35 & 7167.5 & 3290 \\
\hline \multirow{3}{*}{ Model 4 } & 1 & 6100 & 3100 & 2.7 & 16,470 & 8370 \\
& 2 & 4200 & 2500 & 2.7 & 11,340 & 6750 \\
& 3 & 3050 & 1600 & 2.7 & 8235 & 4320 \\
\hline
\end{tabular}
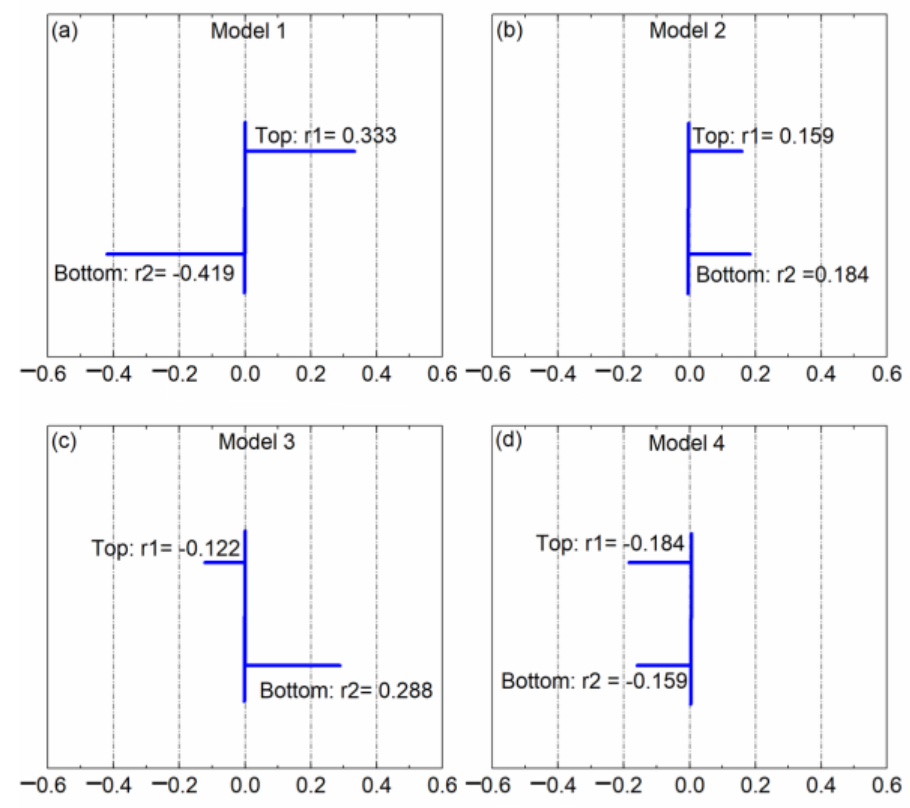

Figure 2. Reflectivity logs of Models 1-4 for normal incidence: (a) Model 1, (b) Model 2, (c) Model 3 and (d) Model 4.

For a better understanding of the behavior of the linear analytic approximate formula, we compare the linear analytic approximation and the true value of thin-bed P-wave reflection coefficients [20] by illustrating $\lambda / 10$ and $\lambda / 20$ examples, as shown in Figure 3. In order to prove that the parameters $P$ and $\mathrm{G}$ are derived accurately, the cases of thin-bed thicknesses equal to zero, i.e., single-interface cases, are also shown in Figure 3. The absolute values of relative errors are plotted in Figure 4. Thin-bed PP-wave reflection coefficients are complex valued. Therefore, we discuss thin-bed P-wave reflection coefficients in terms of their amplitude and phase sections, which are shown in Figures 3 and 4. 

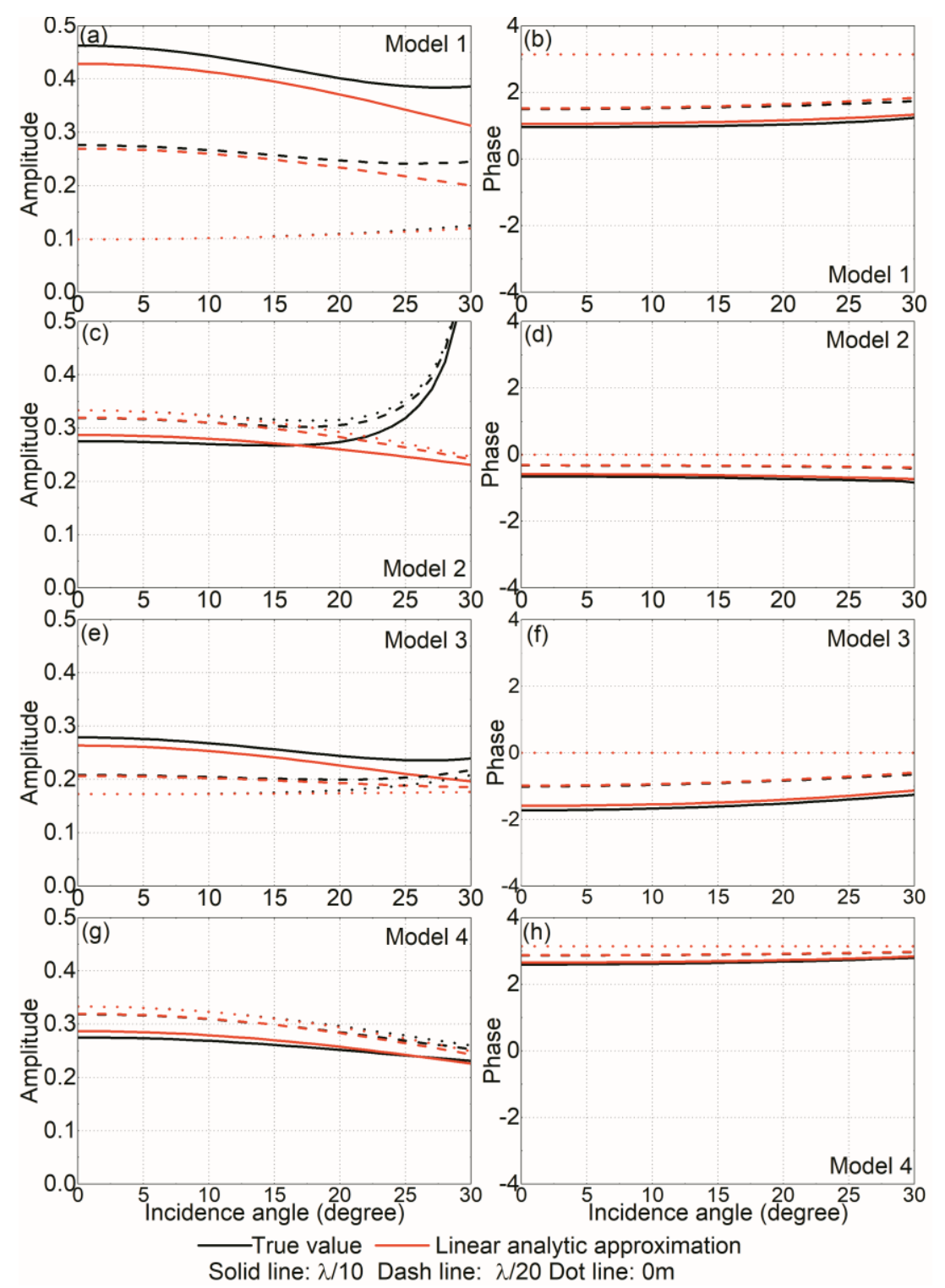

Figure 3. Comparison of the linear analytic approximation and the true value of P-wave reflection coefficients: (a) amplitude of Model 1; (b) phase of Model 1; (c) amplitude of Model 2; (d) phase of Model 2; (e) amplitude of Model 3; (f) phase of Model 3; (g) amplitude of Model 4; (h) phase of Model 4.

Figure 3 shows the P-wave reflection coefficients as a function of incidence angle for Models 1-4 for only three of the modeled cases, $\lambda / 10, \lambda / 20$ and $0 \mathrm{~m}$. As models with opposite-polarity reflections, Models 1 and 3 share similar regularity. In the angle range from 0 to 20 degrees, the linear analytic approximation demonstrates the same AVA gradient, but a lower AVA intercept, compared to the true $\mathrm{PP}$-wave response. For incidence angles greater than 20 degrees, the linear analytic approximation starts to diverge further from the true value. An explanation for this behavior is that the linear analytic approximation relies on the small-incidence assumption. As models with identical-polarity reflections, Models 2 and 4 share similar regularity only for incidence angles smaller than 20 degrees. For Model 2 with a 30-degree critical angle, the AVA curves of the linear analytic approximation diverge further from the true value with increasing angle over 20 degrees. Especially near the critical angle, the true P-wave reflection coefficient increases sharply, while the linear analytic approximation becomes invalid. For Model 4 with no critical angles, the linear analytic approximation is nearly the same as the true values. Comparison of the $\lambda / 10$ and $\lambda / 20$ cases shows that a thinner bed results in a higher 
accuracy for amplitude and phase responses of the linear analytic approximation. For single-interface cases, the true equations of thin-bed R/T coefficients are simplified into Zoeppritz equations [20]. Approximate amplitudes are coincident with the true amplitudes for incidence angles less than 20 degrees. Meanwhile, approximate phases are zero or pi, which are equal to the true phases. The high accuracy of approximate values for single-interface cases confirms that derivations of the parameters $\mathrm{P}$ and $\mathrm{G}$ are accurate.
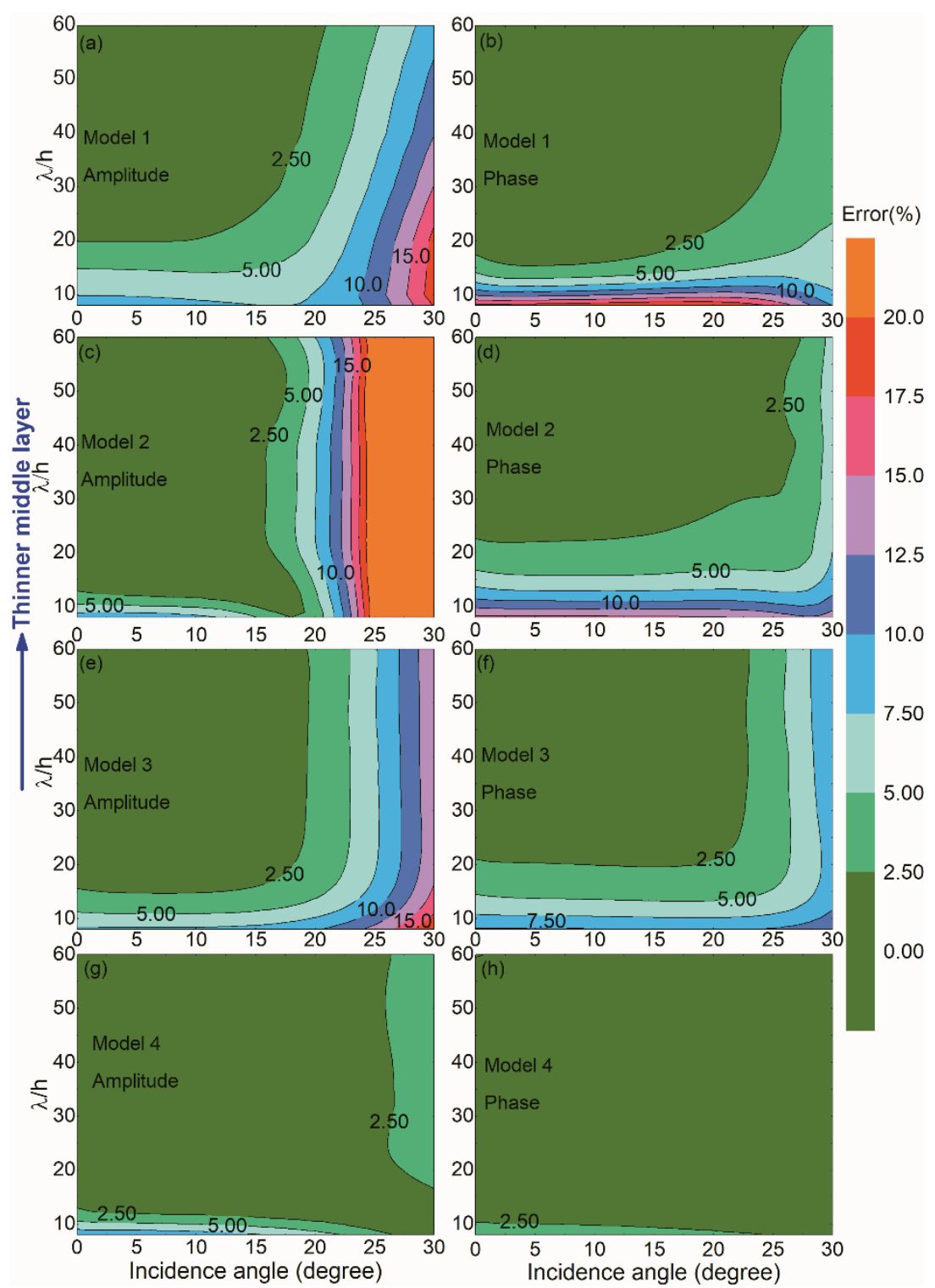

Figure 4. Absolute values of relative errors of thin-bed P-wave reflection coefficients caused by the linear analytic approximation: (a) amplitude of Model 1; (b) phase of Model 1; (c) amplitude of Model 2; (d) phase of Model 2; (e) amplitude of Model 3; (f) phase of Model 3; (g) amplitude of Model 4; (h) phase of Model 4.

Figure 4 shows errors in amplitude (left) and phase (right) caused by the linear analytic approximation for Models 1-4 with incidence angles for the full range of $\lambda / h$ cases tested. Comparison between different $\lambda / \mathrm{h}$ cases for small incidence shows that approximation errors decline with a thinner bed and drop below $5 \%$ rapidly with decreasing thickness of the thin bed. For models with critical angles, i.e., Models 1-3, the relative errors versus incidence angle and thickness share a similar phenomenon. For incidence angles less than 20 degrees and thicknesses less than $\lambda / 10$, the amplitude 
errors are less than 10\%. For incidence angles greater than 20 degrees, the amplitude errors are larger than $10 \%$, and increase with increasing incidence angle and thin-bed thickness. The amplitude errors rise quickly for incidence angles near the critical angles, and the linear analytic approximation becomes invalid. Applicability of the approximate formula for Model 1 shows that the linear analytic approximation is valid for thin-bed models with strong impedance contrasts. For model with no critical angles, i.e., Model 4 , approximation errors smaller than $5 \%$ for a thickness smaller than $\lambda / 8$. Compared with the Model 1-3 cases, the linear analytic approximation for Model 4 has a relatively higher accuracy, which exhibits a better applicability of the linear analytic approximation for low-to-high impedance transition layers.

From numerical tests, we can conclude that: when incidence angles are less than 20 degrees and thin-bed thicknesses are less than one-tenth of the P-wave wavelength, the approximation amplitude errors are smaller than $10 \%$, which verifies the applicability of the linear analytic approximation.

\subsection{Application Examples}

Using the linear analytic approximation of thin-bed P-wave reflection coefficients, thin-bed elastic parameters and thickness are inversed through a least-squares approach. We apply the proposed inversion flow to predict thin-bed properties from synthetic seismic data.

Considering that thin-bed properties of Model 3 are close to those of some actual reservoirs, such as coal seams and unconsolidated sandstone, we take Model 3 as a thin-bed model to exhibit application of the approximate formula to synthetic data in this section. Using the true P-wave reflection coefficient, we simulate the synthetic AVA records of Model 3 using the Ricker wavelet with a $25 \mathrm{~Hz}$ predominant frequency. The thin-bed thickness is set as $3 \mathrm{~m}$ and the incidence angles range from 0 to 20 degrees. Taking the synthetic AVA records as the real seismic traces, we construct complex seismic traces through the Hilbert transform, which are shown in Figure 5. Compared with the real component, the imaginary records have the same amplitude envelope but 90-degree phase shift. Thin-bed complex reflection coefficients are derived using Equation (10).

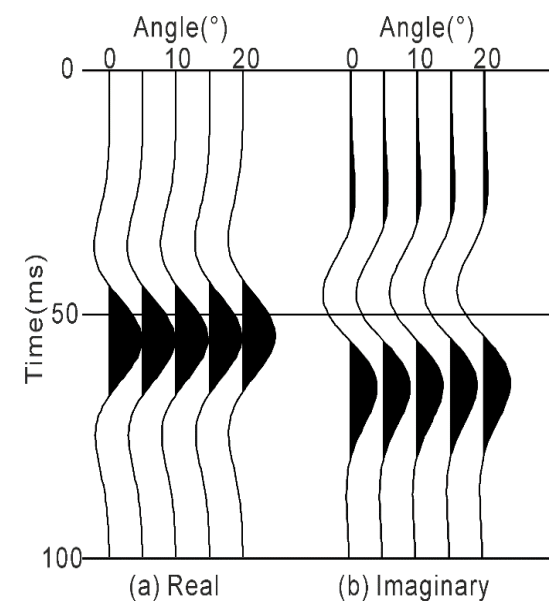

Figure 5. Complex seismic traces of Model 3: (a) real component; (b) imaginary component.

The calculation procedure of thin-bed reflection coefficients is shown in Figure 6 by taking normal responses of Model 3 as an example. Using the real and imaginary reflection coefficients, we invert for thin-bed properties by the proposed thin-bed inversion method and by the conventional AVA inversion method based on the Zoeppritz equations. As shown in Figure 7, the proposed thin-bed inversion method is superior in the characterization of thin-bed properties compared to the conventional AVA inversion method. The proposed thin-bed inversion method can derive more accurate elastic parameters of the target thin bed and its upper and lower half spaces. In particular, the inversion results can indicate the thickness of the target thin bed clearly and accurately. 

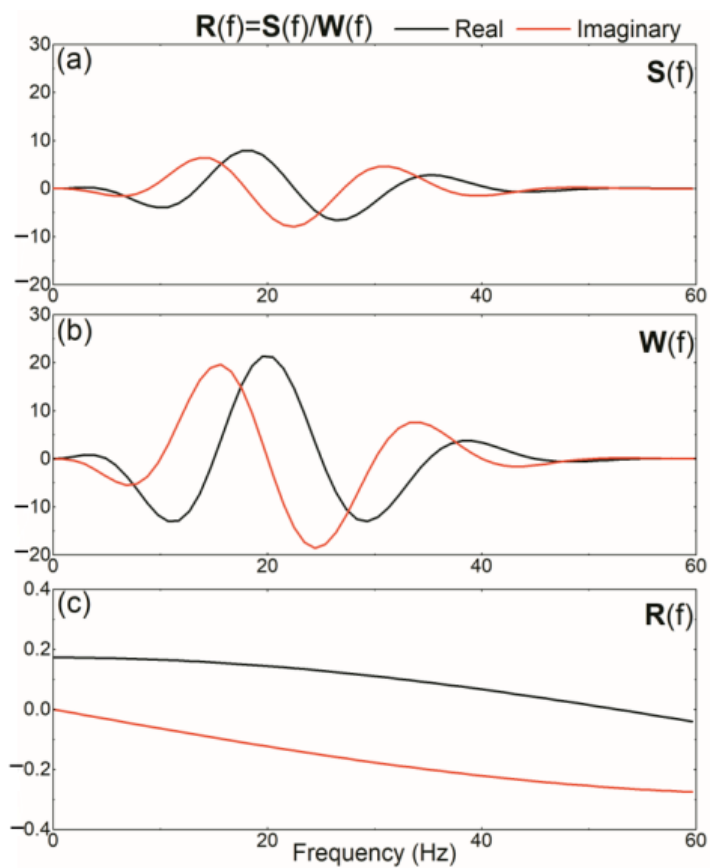

Figure 6. Example of Model 3 at normal incidence for $R(\theta, f)=S(\theta, f) / W(\theta, f)$ : (a) real and imaginary components of seismic records; (b) real and imaginary components of Ricker wavelet; (c) real and imaginary components of thin-bed P-wave reflection coefficients.

$\begin{array}{lll}\text { (a) P-wave velocity }(\mathrm{km} / \mathrm{s}) & \text { (b) S-wave velocity }(\mathrm{km} / \mathrm{s})\end{array}$

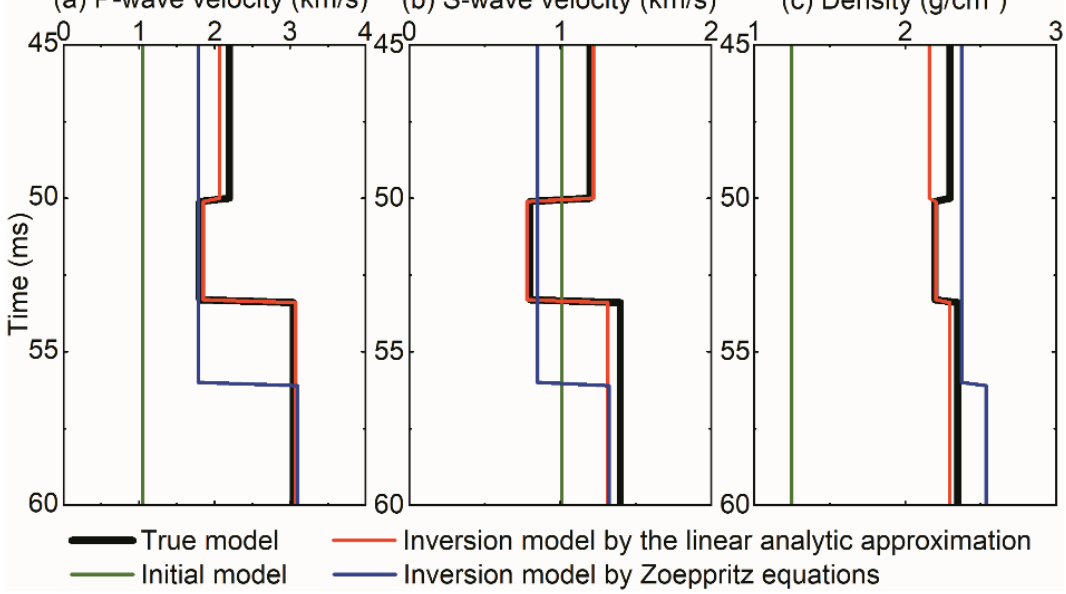

Figure 7. Inversion results of Model 3 by the linear analytic approximation and Zoeppritz equations: (a) P-wave velocity; (b) S-wave velocity; (c) density.

In order to test the influence of noise on the inversion, we add $10 \%$ (mean energy ratio) and $20 \%$ white noise to the real AVA records, which are shown in Figure 8. 


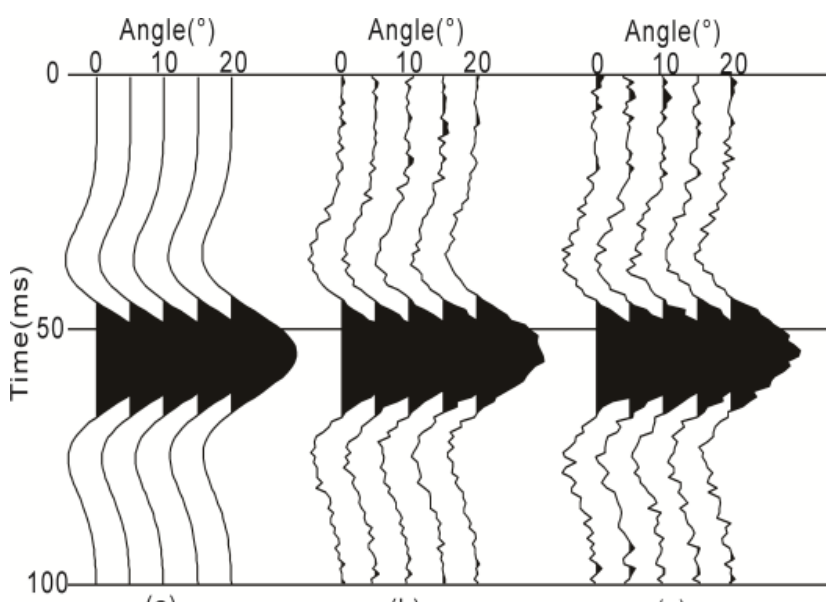

(a)

(b)

(c)

Figure 8. Seismic traces of Model 3: (a) 0\% noise; (b) 10\% white noise; (c) 20\% white noise.

The inverted results of thin-bed properties are shown in Figure 9. For $10 \%$ white noise data, it can be seen that thin-bed thickness is basically coincident with that of the true model. The inverted P-wave and S-wave velocities deviate from the true model relatively more. Compared with the P-wave and $\mathrm{S}$-wave velocities, the error in the density inversion is larger. The reason is that incidence angles of our AVA gather are from 0 to 20 degrees, which are not so large. Normally, stable density inversion requires larger incidence angle gathers. Compared to the $10 \%$ white noise case, the inverted P-wave and S-wave velocities for the $20 \%$ white noise case have higher inversion errors, while they can reveal the true size relationship of thin-bed elastic parameters.

(a) P-wave velocity $(\mathrm{km} / \mathrm{s})$

(b) S-wave velocity $(\mathrm{km} / \mathrm{s})$

(c) Density $\left(\mathrm{g} / \mathrm{cm}^{3}\right)$

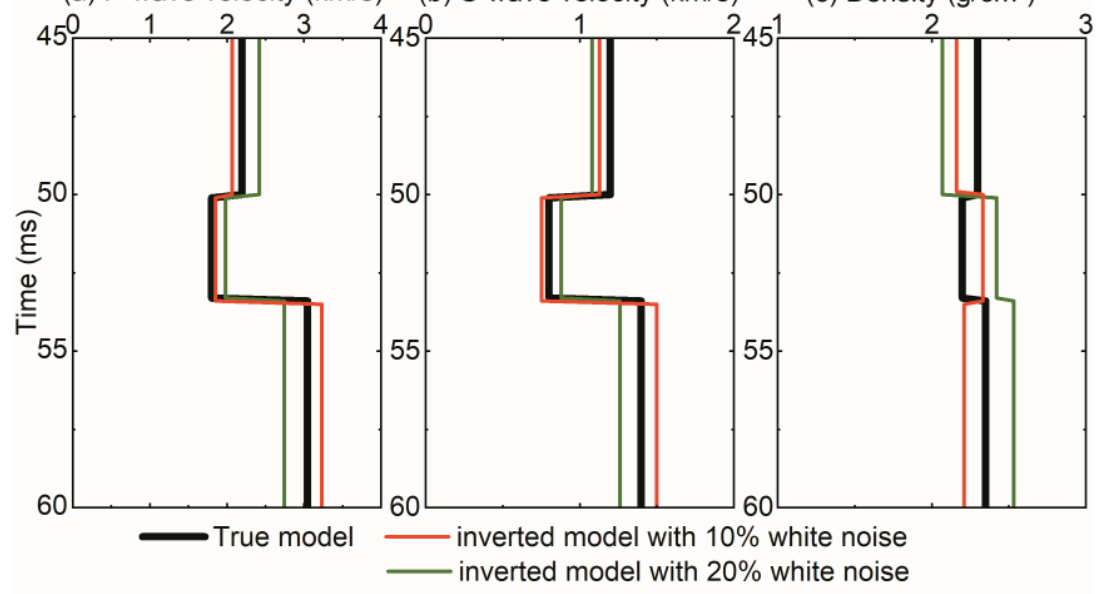

Figure 9. Inversion results of Model 3's amplitude versus angle (AVA) records with $10 \%$ and $20 \%$ white noise by the linear analytic approximation: (a) P-wave velocity; (b) S-wave velocity; (c) density.

In order to test the thin-bed inversion strategy against synthetic data of multi-layer models, we set a six-layer 1D model named "Model 5", and synthetic P-wave AVA datasets, as shown in Figure 10. Table 2 lists the elastic parameters and layer thicknesses of Model 5. A Ricker wavelet with a $25 \mathrm{~Hz}$ predominant frequency is chosen as the synthetic seismic wavelet.

Under the seismic resolution limit of a $25 \mathrm{~Hz}$ predominant frequency, Model 5 includes an impedance interface and two thin beds. The impedance interface is between layer 1 and layer 2 , and is a low-to-high impedance interface. One thin bed is a $3 \mathrm{~m}$ low-impedance thin bed, which is constituted by layer 2, layer 3 and layer 4 . The other thin bed is a $6 \mathrm{~m}$ low-to-high impedance transition layer, 
which is constituted by layer 4 , layer 5 , and layer 6 . Figure 11 shows the inversion results of thin-bed properties by the proposed thin-bed inversion and those by the conventional AVA inversion [34].

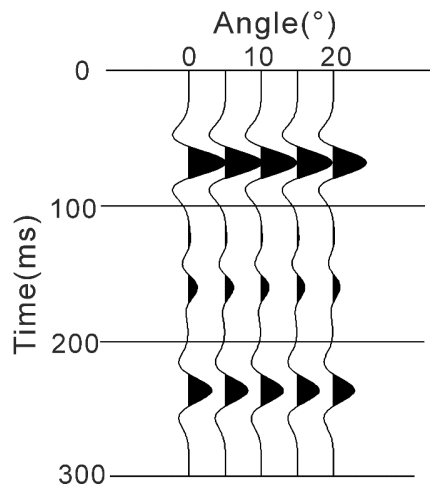

Figure 10. PP-wave AVA gathers of Model 5.

Table 2. Parameters of multi-layer models. Units of velocities, densities and thickness in Table 2 are $\mathrm{m} / \mathrm{s}, \mathrm{g} / \mathrm{cm}^{3}$ and $\mathrm{m}$, respectively.

\begin{tabular}{cccccc}
\hline & Layer No. & $\boldsymbol{v}_{\boldsymbol{P}}$ & $\boldsymbol{v}_{\boldsymbol{S}}$ & $\boldsymbol{\rho}$ & Thickness \\
\hline & 1 & 1500 & 700 & 1.80 & 50 \\
Model 5 & 2 & 2400 & 1200 & 2.31 & 100 \\
& 3 & 1800 & 800 & 2.20 & 3 \\
& 4 & 2500 & 1250 & 2.32 & 100 \\
& 5 & 3000 & 1500 & 2.40 & 6 \\
& 6 & 3500 & 1700 & 2.60 & $\infty$ \\
\hline
\end{tabular}

(a) P-wave velocity $(\mathrm{km} / \mathrm{s})$ (b) S-wave velocity $(\mathrm{km} / \mathrm{s})$ (c) Density $\left(\mathrm{g} / \mathrm{cm}^{3}\right)$

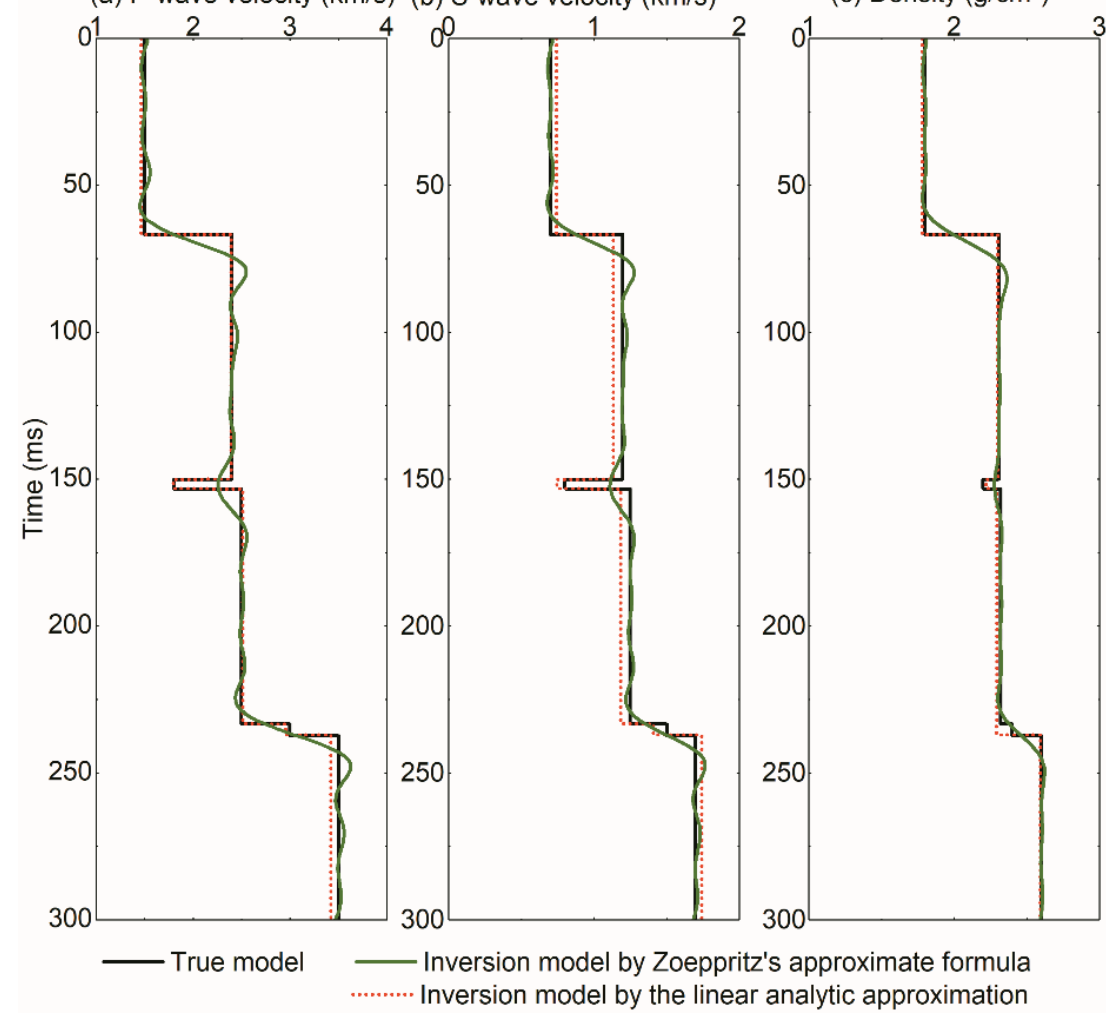

Figure 11. Inversion results of Model 5 by the linear analytic approximation and Zoeppritz's approximate formula: (a) P-wave velocity; (b) S-wave velocity; (c) density. 
For the impedance interface located at $67 \mathrm{~ms}$ in the time domain, the inversion results by the proposed thin-bed inversion show that the middle layer is equal to the third layer. Meanwhile, the inverted P-wave velocities and densities are very close to the true P-wave velocities and densities, respectively. Compared with the inverted results by Zoeppritz's approximate formula, the inverted $\mathrm{S}$-wave velocities diverge relatively further from the true S-wave velocities. Meanwhile, considering that thin-bed inversion needs to carry out complicated calculations in a complex domain, conventional Zoeppritz inversion is a better choice for an impedance interface. For the low-impedance thin bed located at $150 \mathrm{~ms}$, compared with the conventional AVA inversion, the proposed thin-bed inversion method derives more accurate elastic parameters of the target thin bed. For the low-to-high impedance transition layers located at $233 \mathrm{~ms}$, the conventional AVA inversion method cannot reveal the existence of the target thin bed. Meanwhile, the thin-bed inversion reveals the existence of the target thin bed very well. Moreover, the inverted P-wave velocities and thin-bed thicknesses are close to the true values.

\section{Discussion}

Compared with the reflection coefficient equations of a single interface, the thin-bed reflectivity approximation gives closer descriptions of thin-bed seismic responses. However, the approximate formula is also based on the fundamental assumptions of conventional AVO methods, such as horizontal interface, plane-wave incidence, etc. Therefore, the approximate formula is more applicable for the thin-bed reservoirs with relatively simple structures. For the inversion problems of complicated structure reservoirs, the inversion methods based on numerical solutions of wave equation are being studied and applied in depth. For example, recently, Pan et al. [35] successfully applied elastic full-waveform inversion to a field dataset for reservoir characterization. However, at present, the published papers on the thin-bed inversion based on numerical solutions of wave equation are very rare. Therefore, the approximate formula and the corresponding inversion method proposed in this paper still has potentially exploitable superiority for thin-bed AVO inversion.

This paper concentrates on the P-wave reflectivity approximation of a single thin bed. The situation becomes more complex if the target thin layer is embedded in a finely layered background. Research on finely layered reservoirs shows that the interlayer structure and lithologies can be generally determined through long-wavelength approximation [36-41]. Therefore, we will introduce long-wavelength approximation in our further AVA inversion of finely layered reservoirs.

The proposed thin-bed inversion strategy inverts for ten parameters of a thin bed, including P-wave and S-wave velocities and densities of the target thin bed and its upper and lower half spaces and thin-bed thickness. Thin-bed AVA inversion is prone to obtaining local minimum solutions due to many unknown parameters to invert. In this paper, we adopt logging information to constrain the inverted thin-bed model for reasonable inversion results. Studies on a single interface show that the parameterization method reduces the number of unknown parameters and improves the stability of single-interface inversion [42,43]. Therefore, we will introduce the parameterization method in our further AVA inversion of a thin bed for a more stable inversion.

In the tests of synthetic seismic data, we just made a preliminary attempt to do the inversion based on our formula. The noise tests show that the inversion method has a certain anti-noise ability. Moreover, we have not yet developed a mature process and program for the large-scale industrial applications of our inversion method. At present, we can only build the initial model manually and invert the AVA gathers one by one. In a future study, we will try to enhance the anti-noise capabilities of the inversion method, and develop matching data processing techniques.

\section{Conclusions}

This paper derives thin-bed P-wave reflectivity as a linear analytic approximation under small-incidence and thin-bed assumptions. The linear analytic approximation can be reduced to formulas in case of a single interface when the thin-bed thickness is set to zero. Numerical tests of 
four representative thin-bed models show that when incidence angles are less than 20 degrees and thin-bed thicknesses are less than one-tenth of the P-wave wavelength, the approximated amplitude errors are smaller than $10 \%$. Compared to the second-order series approximation, the linear analytic approximation has a relatively compact form, which helps facilitate usage in an inversion scheme.

Using the linear analytic approximation, this paper proposes an inversion methodology of thin-bed properties (P-wave velocities, S-wave velocities, densities of the target thin bed and its overburden and underburden, as well as thin-bed thickness) from AVA gathers through a least-squares algorithm. Inversion applications to synthetic data exhibit the applicability of the linear analytic approximation in thin-bed AVA inversion.

Author Contributions: Conceptualization, C.Y. and Y.W.; methodology, C.Y. and Y.W.; software, C.Y. and S.X.; validation, C.Y. and S.X.; formal analysis, C.Y., Z.L. and H.H.; writing-original draft preparation, C.Y.; writing-review and editing, C.Y. and Y.W.; funding acquisition, Y.W. All authors have read and agreed to the published version of the manuscript.

Funding: This research was funded by the 13th Five-year Plan Project Program "seismic wave characteristics and inversion of a thin layer (2016ZX05002-005-008)", National Natural Science Foundation of China (U1910205), the Fundamental Research Funds for the Central Universities, China (2652019004).

Acknowledgments: The authors would like to thank the anonymous reviewers for their constructive comments and suggestions.

Conflicts of Interest: The authors declare no conflict of interest.

\section{Appendix A}

In Equation (1), $A_{0}$ and $A_{2}$ are defined as

$$
A_{0}=\frac{1}{m_{0}}\left[\left(\frac{z_{P 3}}{z_{P 1}}-1\right) \cos \tau+j\left(\frac{z_{P 2}}{z_{P 1}}-\frac{z_{P 3}}{z_{P 2}}\right) \sin \tau\right]
$$

and

$$
\begin{gathered}
A_{2}=\frac{1}{m_{0}}\left[-\frac{l_{1}^{2}}{2} \tau \sin \tau+2 \frac{a_{3} l_{1}^{2}}{r_{2}^{2}}\left(d_{1}-1\right)+\frac{2}{r_{1}^{2}} \cos \tau+b_{1} \frac{z_{P 3}}{z_{P 1}}+j b_{3} \frac{z_{P 3}}{z_{P 2}}\right]\left(1+A_{0}\right) \\
+\frac{1}{m_{0}}\left(b_{6} \frac{z_{P 3}}{z_{P 1}}+\frac{2 j b_{2} l_{1}}{r_{1}^{2}}+j b_{4} \frac{z_{P 2}}{z_{P 1}}\right)\left(1-A_{0}\right)+\frac{b_{5}}{m_{0}}\left[l_{1} l_{2}\left(\frac{r_{3}^{2}}{4}-1\right) B_{0}+\left(\frac{r_{3}}{2}-1\right) B_{1}\right] \\
+\frac{1}{m_{0}}\left[\begin{array}{c}
\left(b_{1}+\frac{\cos \tau}{r_{1}}\right) \frac{z_{P 3}}{z_{P 1}}+\frac{2}{r_{1}^{2}} \cos \tau+l_{1} a_{3}\left(\frac{2}{r_{2}^{2}} \frac{z_{P 2}}{z_{P 1}}+\frac{1}{r_{1}} \frac{z_{P 3}}{z_{P 2}}\right) \\
+\frac{2 j \sin \tau}{r_{1}^{2}} \frac{z_{P 3}}{z_{P 2}}+j \frac{\sin \tau}{r_{1}} \frac{z_{P 2}}{z_{P 1}}-j \frac{b_{2} l_{1}}{r_{1}}
\end{array}\right] A_{1}
\end{gathered}
$$

where

$$
\begin{gathered}
B_{0}=\frac{2}{m_{0}} \\
A_{1}=\frac{1}{m_{1}}\left[\left(\frac{a_{3}}{d_{1}}-\cos q\right) \frac{z_{S 3}}{z_{S_{1} 1}}+j\left(a_{5}-\frac{z_{S 2}}{z_{S 1}} \sin q\right)\right]\left(1+A_{0}\right)+\frac{1}{m_{1}}\left(l_{1} l_{2} \frac{z_{S 3}}{z_{S 1}}-a_{4}\right) B_{0} \\
+\frac{1}{m_{1}}\left[\left(\frac{1}{r_{1}}-\frac{l_{1}}{r_{2}} \frac{z_{S 2}}{z_{S 1}}\right) a_{6}+\frac{2 l_{1}}{r_{2}} \cos \tau \frac{z_{S 2}}{z_{S 1}}+j l_{1} \sin \tau \frac{z_{S 3}}{z_{S_{S 1}}}\right]\left(1-A_{0}\right)
\end{gathered}
$$

and

$$
\begin{aligned}
& B_{1}=\frac{1}{m_{1}}\left(1-\frac{a_{2} a_{3}}{d_{1}}+j a_{1} a_{5}\right)\left(1+A_{0}\right)-\frac{1}{m_{1}}\left[l_{1} l_{2} a_{2}+(\cos q+j \sin q) a_{4}\right] B_{0} \\
& +\frac{1}{m_{1}}\left[\frac{2}{r_{1}}-j a_{2} l_{1} \sin \tau+\frac{2 l_{1}}{r_{2}} \frac{z_{S 2}}{z_{\mathcal{S} 1}}\left(a_{1} \cos \tau-1\right)\right]\left(1-A_{0}\right)
\end{aligned}
$$

with

$$
\begin{gathered}
m_{0}=\left(z_{P 3} / z_{P 1}+1\right) \cos \tau+j\left(z_{P 2} / z_{P 1}+z_{P 3} / z_{P 2}\right) \sin \tau, \\
m_{1}=\left(z_{S 3} / z_{S 1}+1\right) \cos q+j\left(z_{S 2} / z_{S 1}+z_{S 3} / z_{S 2}\right) \sin q, \\
a_{1}=\cos q+j \frac{z_{S 1}}{z_{S 2}} \sin q, a_{2}=\cos q+j \frac{z_{S 2}}{z_{S 1}} \sin q, a_{3}=\cos q-\cos \tau, \\
a_{4}=\frac{2 l_{1} l_{2}}{r_{3}} \frac{z_{S 3}}{z_{S 1}}, a_{5}=\frac{r_{1} l_{1}}{r_{2}}\left(\sin q-\frac{2}{r_{2}} \sin \tau\right), a_{6}=2\left(\cos q+j \sin q \frac{z_{S 3}}{z_{S 2}}\right),
\end{gathered}
$$




$$
\begin{gathered}
b_{1}=\frac{j l_{1}}{r_{2}}\left(\sin q-\frac{2}{r_{2}} \sin \tau\right), b_{2}=\sin \tau-\frac{2}{r_{2}} \sin q, \\
b_{3}=\frac{l_{1}^{2}}{2}(\sin \tau+\tau \cos \tau)-\frac{l_{1}^{2}}{r_{2}} \sin q+\frac{2}{r_{1}^{2}} \sin \tau, \\
b_{4}=4 \frac{l_{1}^{2}}{r_{2}^{2}}\left(\frac{1}{r_{2}} \sin q-\sin \tau\right)+\frac{l_{1}^{2}}{2}(\sin \tau-\tau \cos \tau)-\frac{1}{2} \sin \tau, \\
b_{5}=\frac{2 l_{1} l_{2}}{r_{3}^{2}} \frac{z_{P 3}}{z_{P 1}}, b_{6}=\frac{1}{2}\left(l_{1}^{2} \tau \sin \tau-\cos \tau\right)+2 a_{3}\left(\frac{l_{1}^{2}}{r_{2}^{2}}-\frac{1}{r_{1}^{2} d_{1}}\right), \\
\tau=\frac{\omega h}{v_{P 2}}, q=\frac{\omega h}{v_{S 2}}, d_{1}=\frac{\rho_{2}}{\rho_{1}},
\end{gathered}
$$

where $z_{P}=\rho v_{\mathrm{P}}$ are P-wave impedances, $z_{S}=\rho v_{\mathrm{S}}$ are S-wave impedances, $r=v_{\mathrm{P}} / v_{\mathrm{S}}$ are the ratios of P-wave velocities to $S$-wave velocities and their subscripts 1, 2, 3 refer to three layers of the single thin bed, respectively. $l_{\mathrm{k}}=v_{\mathrm{Pk}+1} / v_{\mathrm{Pk}}$ are the ratios of the P-wave velocities in adjacent layers with $k=1,2$, respectively; $v_{P}$ and vs. are P-wave and S-wave velocities, respectively, $\rho$ is density, $\delta$ is S-wave transmitted or reflected angle and their subscripts 1, 2, 3 refer to three layers of the thin bed, respectively; $\theta$ is P-wave incidence angle, $\theta_{2}$ and $\theta_{3}$ are P-wave transmitted or reflected angle in the target thin bed and its floor, respectively; $h$ is the thickness of the middle layer, $\omega$ is angular frequency and $j$ is the imaginary symbol.

\section{Appendix B}

In Equation (3), there are

$$
\begin{aligned}
A=\frac{1}{n_{1}} & \left(c_{1}-\frac{z_{S 3}}{z_{S 1}}-j \tau r_{2} \frac{z_{S 2}}{z_{S 1}}\right)(1+P)+\frac{l_{1} l_{2}}{n_{1}}\left(1-\frac{2}{r_{3}}\right) \frac{z_{S 3}}{z_{S 1}} \frac{2}{n_{0}}\left(1+\tau^{2}\right), \\
& +\frac{1}{n_{1}}\left[\frac{2}{r_{1}}+j \tau\left(-l_{1} \frac{z_{S 3}}{z_{S 1}}+2 \frac{r_{2}}{r_{1}} \frac{z_{S 3}}{z_{S 2}}\right)\right](1-P)
\end{aligned}
$$

and

$$
\begin{aligned}
B=\frac{1}{n_{1}} & {\left[c_{2}+c_{1}\left(1+j \tau r_{2} \frac{z_{S 1}}{z_{S 2}}\right)\right](1+P) } \\
& +\frac{1}{n_{1}}\left[\frac{2 c_{2}}{r_{1}}+j \tau l_{1}\left(1+j \tau r_{2} \frac{z_{S 2}}{z_{S 1}}\right)\right](1-P) \\
& -\frac{l_{1} l_{2}}{n_{1}}\left[1+\frac{2}{r_{3}} \frac{z_{S 3}}{z_{S 1}}+j \tau r_{2}\left(\frac{\left(S_{S 2}\right.}{z_{S 1}}+\frac{2}{r_{3}} \frac{z_{S 3}}{z_{S 2}}\right)\right] \frac{2}{n_{0}}\left(1+\tau^{2}\right)
\end{aligned}
$$

with $n_{0}=\left(\frac{z_{P 3}}{z_{P 1}}+1\right)+j \tau\left(\frac{z_{P 2}}{z_{P 1}}+\frac{z_{P 3}}{z_{P 2}}\right), \quad n_{1}=\left(\frac{z_{S 3}}{z_{S 1}}+1\right)+j \tau r_{2}\left(\frac{z_{S 3}}{z_{S_{2}}}+\frac{z_{S 2}}{z_{S 1}}\right), \quad c_{1}=j \tau r_{1} l_{1}\left(1-\frac{2}{r_{2}^{2}}\right)$ and $c_{2}=1+\tau^{2} r_{2}^{2}$.

\section{References}

1. Zeng, H.; Backus, M.M. Interpretive advantages of $90^{\circ}$-phase wavelets: Part 1-Modeling. Geophysics 2005, 70, C7-C15. [CrossRef]

2. Almoghrabi, H.; Lange, J. Layers and bright spots. Geophysics 1986, 51, 699-709. [CrossRef]

3. Juhlin, C.; Young, R. Implications of thin layers for amplitude variation with offset (AVO) studies. Geophysics 1993, 58, 1200-1204. [CrossRef]

4. Widess, M.B. How thin is a thin bed? Geophysics 1973, 38, 1176-1180. [CrossRef]

5. Neidell, N.S.; Poggiagliolmi, E. Stratigraphic modeling and interpretation-Geophysical principles, in Payton, C.E., Eds., Seismic stratigraphy-Application to hydrocarbon exploration. Am. Assn. Petr. Geol. Memoir $1977,26,389-416$.

6. Koefoed, O.; De Voogd, N. The linear properties of thin layers, with an application to synthetic seismograms over coal seams. Geophysics 1980, 45, 1254-1268. [CrossRef]

7. De Voogd, N.; Rooijen, H.D. Thin-layer response and spectral bandwidth. Geophysics 1983, 48, 12-18. [CrossRef] 
8. Chung, H.; Lawton, D.C. Amplitude responses of thin beds: Sinusoidal approximation versus Ricker approximation. Geophysics 1995, 60, 223-230. [CrossRef]

9. Chung, H.M.; Lawton, D.C. Frequency characteristics of seismic reflections from thin beds. Can. J. Explor. Geophys. 1995, 31, 32-37.

10. Liu, Y.; Schmitt, D.R. Amplitude and AVO responses of a single thin bed. Geophysics. 2003, 68, 1161-1168. [CrossRef]

11. Thomson, W.T. Transmission of Elastic Waves through a Stratified Solid Medium. J. Appl. Phys. 1950, 21, 89-93. [CrossRef]

12. Haskell, N.A. The dispersion of surface waves on multilayered media. In Vincit Veritas: A Portrait of the Life and Work of Norman Abraham Haskell; Wiley: New Jersey, NJ, USA, 1990; pp. 86-103. [CrossRef]

13. Brekhovskikh, L.M. Waves in Layered Media; Academic Process: Tamil Nadu, India, 1960.

14. Meissner, R.; Meixner, E. Deformation of seismic wavelets by thin layers and layered boundaries. Geophys. Prospect. 1969, 17, 1-27. [CrossRef]

15. Yang, C.; Wang, Y.; Lu, J. Weak impedance difference approximations of thin-bed PP-wave reflection responses. J. Geophys. Eng. 2017, 14, 1010-1019. [CrossRef]

16. Kennett, B.L.N.; Kerry, N.J.; Woodhouse, J.H. Symmetries in the reflection and transmission of elastic waves. Geophys. J. Int. 1978, 52, 215-229. [CrossRef]

17. Kennett, B.L.N. Seismic Wave Propagation in a Stratified Medium; Cambridge University Press: Cambridge, UK, 1983.

18. Rubino, J.G.; Velis, D. Thin-bed pre-stack spectral inversion. Geophysics 2009, 74, R49-R57. [CrossRef]

19. Pan, W.; Innanen, K.A. AVO/AVF analysis of thin beds in elastic media. SEG Tech. Program Expand. Abstr. 2013, 2013, 373-377.

20. Yang, C.; Wang, Y.; Wang, Y. Reflection and transmission coefficients of a thin bed. Geophysics 2016, 81, N31-N39. [CrossRef]

21. Yang, C.; Wang, Y.; Lu, J.; Chen, B.; Shi, L. A Low-Order Series Approximation of Thin-Bed PP-Wave Reflections. Appl. Sci. 2019, 9, 709. [CrossRef]

22. Aki, K.; Richards, P.G. Quantitative Seismology_Theory and Methods; W.H. Freeman and Co: New York, NY, USA, 1980.

23. Shuey, R.T. A simplification of the Zoeppritz equations. Geophysics 1985, 50, 609-614. [CrossRef]

24. Smith, G.; Gidlow, P. Weighted stacking for rock property estimation and detection of gas. Geophys. Prospect. 1987, 35, 993-1014. [CrossRef]

25. Taner, M.T.; Koehler, F.; Sheriff, R.E. Complex seismic trace analysis. Geophysics 1979, 44, 1041-1063. [CrossRef]

26. Robertson, J.D.; Nogami, H.H. Complex seismic trace analysis of thin beds. Geophysics 1984, 49, $344-352$. [CrossRef]

27. Barnes, A.E. Theory of 2-D complex seismic trace analysis. Geophysics 1996, 61, 264-272. [CrossRef]

28. Liu, Y.; Tinivella, U.; Liu, X. An inversion method for seafloor elastic parameters. Geophysics 2015, 80, N11-N21. [CrossRef]

29. Levenberg, K. A method for the solution of certain non-linear problems in least squares. Q. Appl. Math. 1944, 2, 164-168. [CrossRef]

30. Marquardt, D.W. An algorithm for least-squares estimation of non-linear inequalities. J. Soc. Ind. Appl. Math. 1963, 11, 431-441.

31. Lu, J.; Yang, Z.; Wang, Y.; Shi, Y. Joint PP and PS AVA seismic inversion using exact Zoeppritz equations. Geophysics 2015, 80, R239-R250. [CrossRef]

32. Lu, J.; Wang, Y.; Chen, J. Detection of Tectonically Deformed Coal Using Model-Based Joint Inversion of Multi-Component Seismic Data. Energies 2018, 11, 829. [CrossRef]

33. Chen, T.; Liu, Y. Multi-component AVO response of thin beds based on reflectance spectrum theory. Appl. Geophys. 2006, 3, 27-36. [CrossRef]

34. Fatti, J.L.; Smith, G.C.; Vail, P.J.; Strauss, P.J.; Levitt, P.R. Detection of gas in sandstone reservoirs using AVO analysis: A 3-D seismic case history using the Geostack technique. Geophysics 1994, 59, 1362-1376. [CrossRef]

35. Pan, W.; Innanen, K.A.; Geng, Y. Elastic full-waveform inversion and parameterization analysis applied to walk-away vertical seismic profile data for unconventional (heavy oil) reservoir characterization. Geophys. J. Int. 2018, 213, 1934-1958. [CrossRef] 
36. Postma, G.W. Wave propagation in a stratified medium. Geophysics 1955, 20, 780-806. [CrossRef]

37. Backus, G.E. Long-wave elastic anisotropy produced by horizontal layering. J. Geophys. Res. Space Phys. 1962, 67, 4427-4440. [CrossRef]

38. Stovas, A.; Landrø, M.; Avseth, P. AVO attribute inversion for finely layered reservoirs. Geophysics 2006, 71 , C25-C36. [CrossRef]

39. Stovas, A.; Landro, M. Uncertainty in discrimination between net-to-gross and water saturation for fine-layered reservoirs. SEG Tech. Program Expand. Abstr. 2006, 25, 1698-1702. [CrossRef]

40. Roganov, Y.; Stovas, A. Low-frequency wave propagation in periodically layered media. Geophys. Prospect. 2012, 60, 825-837. [CrossRef]

41. An, Y.; Lu, J. Calculation of AVA Responses for Finely Layered Reservoirs. Math. Probl. Eng. 2018, 2018, 1-11. [CrossRef]

42. Lavaud, B.; Kabir, N.; Chavent, G. Pushing AVO Inversion beyond Linearized Approximation. In Proceedings of the EAGE 61st Conference on European Association Geoscience and Engineering, Helsinki, Finland, 7-11 June 1999; pp. 6-49. [CrossRef]

43. Kurt, H. Joint inversion of AVA data for elastic parameters by bootstrapping. Comput. Geosci. 2007, 33, 367-382. [CrossRef]

Publisher's Note: MDPI stays neutral with regard to jurisdictional claims in published maps and institutional affiliations.

(C) 2020 by the authors. Licensee MDPI, Basel, Switzerland. This article is an open access article distributed under the terms and conditions of the Creative Commons Attribution (CC BY) license (http://creativecommons.org/licenses/by/4.0/). 